Endocuff Vision Reduces Inspection Time Without Decreasing Lesion Detection in a Randomized Colonoscopy Trial

Short title: Endocuff Vision and withdrawal time

\author{
Douglas K. Rex, M.D ${ }^{1}$ \\ James E. Slaven ${ }^{2}$ \\ Jonathan Garcia $^{1}$ \\ Rachel Lahr ${ }^{1}$ \\ Meghan Searight ${ }^{1}$ \\ Seth A. Gross, M.D ${ }^{3}$
}
${ }^{1}$ Division of Gastroenterology/Hepatology, Department of Medicine, Indiana University School of Medicine, Indianapolis, Indiana
${ }^{2}$ Department of Biostatistics, Indiana University School of Medicine, Indianapolis, Indiana
${ }^{3}$ Division of Gastroenterology, Tisch Hospital, NYU Langone Medical Center, New York, New York

Author contributions:

Please send correspondence to:

Douglas K. Rex

550 North University Boulevard

Suite 4100

Indianapolis, IN

46202

drex@iu.edu

Rex-study design and drafting of the manuscript

Slaven-statistical support

Garcia, Lahr, Searight-data collection, critical review of the manuscript

Gross-study design, critical revision of the manuscript

COI: Rex and Gross are consultants to Olympus Corp.; Slaven, Garcia, Lahr, Searight have no COIs to disclose

Keywords: Endocuff Vision, colorectal polyps, colonoscopy, withdrawal time, adenoma detection rate.

This work was support by a gift from Scott Schurz and his children to the Indiana University Foundation in the name of Douglas K. Rex.

This is the author's manuscript of the article published in final edited form as:

Rex, D. K., Slaven, J. E., Garcia, J., Lahr, R., Searight, M., \& Gross, S. A. (2019). Endocuff Vision Reduces Inspection

Time Without Decreasing Lesion Detection in a Randomized Colonoscopy Trial. Clinical Gastroenterology and 


\begin{abstract}
:
Background \& Aims: Mucosal exposure devices improve detection of lesions during colonoscopy and have reduced examination times in uncontrolled studies. We performed a randomized trial of Endocuff Vision vs standard colonoscopy to compare differences in withdrawal time (the primary end point). We proposed that Endocuff Vision would allow complete mucosal inspection in a shorter time without impairing lesion detection.
\end{abstract}

Methods: Adults older than 40 years undergoing screening or surveillance colonoscopies were randomly assigned to the Endocuff group ( $n=101,43.6 \%$ women) or the standard colonoscopy group ( $n=99 ; 57.6 \%$ women). One of 2 experienced endoscopists performed the colonoscopies, aiming for a thorough evaluation of the proximal sides of all haustral folds, flexures, and valves in the shortest time possible. Inspection time was measured with a stopwatch and calculated by subtracting washing, suctioning, polypectomy and biopsy times from total withdrawal time.

Results: There were significantly fewer women in the Endocuff arm $(P=.0475)$ but there were no other demographic differences between groups. Mean insertion time with Endocuff was 4.0 min vs 4.4 min for standard colonoscopy $(P=.14)$. Mean inspection time with Endocuff was $6.5 \mathrm{~min}$ vs $8.4 \mathrm{~min}$ for standard colonoscopy $(P<.0001)$. Numbers of adenomas detected per colonoscopy $(1.43$ vs $1.07 ; P=.07)$, adenoma detection rate $(61.4 \%$ vs $52 \% ; P=0.21)$, number of sessile serrated polyps per colonoscopy $(0.27$ vs 0.21 ; $P=0.12)$, and sessile serrated polyp detection rate $(19.8 \%$ vs $11.1 \% ; P=0.09)$ were all higher with Endocuff Vision. Results did not differ significantly when we controlled for age, sex, or race.

Conclusion: In a randomized trial, we found inclusion of Endocuff in screening or surveillance colonoscopies to decrease examination time without reducing lesion detection. ClinicalTrials.gov no.: NCT03361917

KEY WORDS: colonoscopy, colorectal polyp, colon cancer; colorectal

\title{
What You Need to Know
}

Background: We performed a randomized trial of Endocuff Vision vs standard colonoscopy to compare differences in withdrawal time and lesion detection in 200 patients.

Findings: Mean inspection time with Endocuff was 6.5 min vs 8.4 min for standard colonoscopy. Numbers of adenomas detected per colonoscopy, adenoma detection rate, number of sessile serrated polyps per colonoscopy, and sessile serrated polyp detection rate did not differ significantly with vs without Endocuff.

Limitations: The study was performed by 2 examiners with extensive experience with Endocuff Vision.

Implications for patient care: Inclusion of Endocuff in screening or surveillance colonoscopies decreases examination time without reducing lesion detection. 
Introduction

Mucosal exposure devices that have shown promise for improving detection include Endocuff ${ }^{1,2}$, Endocuff Vision ${ }^{3}$, Endorings ${ }^{4}$, and wide angle colonoscopy ${ }^{5}$. In a recent randomized trial, we showed that Endocuff on a high definition colonoscope is a dominant strategy over Endorings and very wide angle colonoscopy, resulting in better detection, and faster insertion $^{2}$.

Another potential avenue of investigation for Endocuff, is whether these devices can speed withdrawal while maintaining stable or improved levels of detection. Video recording studies ${ }^{6}$, demonstrate that high quality colonoscope withdrawal consists of careful inspection of the proximal sides of haustral folds, flexures, and colorectal valves, removal of retained pools of debris and fluid, and adequate colonic distention ${ }^{6}$. Most of the work of withdrawal for high level detectors is the process of exposing the mucosa between haustral folds that could otherwise hide lesions during withdrawal. Devices such as Endocuff can be used to hook and flatten haustral folds, improving mucosal exposure. However, an additional benefit of these devices is to make the process of examining between folds faster.

Studies of mucosal exposure devices have almost uniformly targeted polyp detection as their primary endpoint. In meta-analyses, Endocuff increased the adenoma detection rate (ADR) by an average of $7 \%{ }^{1}$. However, some lines of uncontrolled evidence show that mucosal exposure devices are associated with both significantly better detection and shorter withdrawal time ${ }^{7}$, or numerically better detection and significantly shorter withdrawal time ${ }^{8}$. To our knowledge, no previous randomized trial has specifically targeted withdrawal time as a primary endpoint. 
The issue of how to handle withdrawal time and inspection time (the time spent actually examining the colon after subtraction of washing, suctioning, polypectomy, and biopsy) in controlled trials of colonoscopy is complex. When the primary target of a randomized trial is detection, it may be best to force equal inspection times in the study arms. Thus, in a randomized controlled trial comparing the Third Eye Retroscope to standard colonoscopy, withdrawal times were allowed to vary. The results showed better detection with the Third Eye Retroscope, but withdrawal was also longer ${ }^{9}$. Since longer withdrawal times are associated with better detection and cancer prevention ${ }^{10-12}$, allowing withdrawal time to vary cast uncertainty on the benefits of the Third Eye Retroscope for detection. In some trials such as our recent comparison of Endocuff to Endorings to Full-Spectrum Endoscopy to standard colonoscopy, we forced equal inspection times in the four study arms ${ }^{2}$, in order to avoid this confounding issue.

In the current trial, we specifically chose to study Endocuff Vision without forcing the inspection time to be equal to the control arm. Instead the study colonoscopist tried to perform withdrawal as rapidly as they comfortably could expose hidden mucosa to their satisfaction. The hypothesis was that withdrawal time could be shortened using Endocuff Vision, while simultaneously observing at least no reduction in detection employing Endocuff Vision.

Methods

We performed a randomized controlled clinical trial comparing colonoscopy using Olympus high definition colonoscopes with or without Endocuff Vision (Arc Medical Design Ltd., Leeds, UK). Endocuff Vision is marketed in the United States by Olympus (Olympus America Corp., Center Valley, PA). Neither Arc Medical nor Olympus was involved in the study design or provided any devices or other support for the study, nor did they have access to 
any study data at any time prior to publication of the study. No funding source had any involvement in any aspect of the study. The study was performed at academic outpatient units affiliated with Indiana University School of Medicine and NYU Langone Medical Center. Randomization was performed using a computer generated sequence. The randomization was concealed in opaque numbered envelopes and opened after the informed consent was completed. Patients were enrolled and assigned to the study arms by research assistants at both sites.

The primary endpoint was the inspection time. Inspection time was measured during colonoscopy by a study assistant using a stopwatch. Once the cecum had been intubated, retained material was washed and suctioned. As soon as the cecum was prepared for evaluation, the stopwatch was started. During withdrawal, the stopwatch was stopped anytime a polyp was detected, until its resection and retrieval had been completed and inspection started again. The inspection time stopwatch was also stopped as often as necessary to clean out residual debris and pools of fluid until the colon was again ready for inspection. Assuming a mean inspection time in the control group without Endocuff Vision of approximately 9 minutes, we considered that a reduction in the mean time of 1 minute would be a valuable time savings over a large number of procedures. For the study to have an $80 \%$ power to detect a 1 minute difference in inspection time between the groups, assuming the 2-sided, 2-sample t-test, a 5\% significance level, and standard deviation of 2.5 minutes, a sample size of 200 total patients was needed. The study was approved by the Institutional Review Board at Indiana University Health on November 30, 2017. The trial was listed at clinicaltrials.gov (NCT03361917).

The secondary endpoints were colonoscope insertion time and detection endpoints including adenoma detection rate, adenomas per colonoscopy, sessile serrated polyp detection rate, and sessile serrated polyps per colonoscopy. 
Inclusion criteria included age $\geq 40$ years, and indication for colonoscopy of screening or surveillance colonoscopy.. Patients were excluded if they had a polyposis syndrome, Lynch syndrome, inflammatory bowel disease, diagnostic indication, or a previous resection of the colon. Patients were also excluded if they had known severe diverticular disease, were referred for a previous incomplete colonoscopy, or for a resection of a large colorectal polyp.

Statistical analyses were performed to determine if there were statistically significant differences between the devices using Generalized Estimating Equations (GEEs), which allow for the analysis of repeated measures on the visit level data through the use of the appropriate covariance structure, as well as accounting for site affect. GEE models also allow for the modeling of both continuous and categorical outcomes, through the ability to specify the correct data distributions, which include standard ANCOVA methods, logistic regression models, and skewed continuous data. Models were run both unadjusted and adjusted for age, gender, and race, which were included as covariates from both initial bivariate analyses and clinical relevance. Data were also collapsed to patient level, allowing for analyses of summation and ever/never (i.e. any yes vs. all no) results, using the typical ANCOVA, logistic regression, and Chi-Square analyses. All analytic assumptions were verified and all analyses were performed using SAS v9.4 (SAS Institute, Cary, NC). All authors had access to the study data and reviewed and approved the final manuscript.

Results

There were 200 patients randomized, including 101 to Endocuff Vision and 99 to standard colonoscopy. The mean patient age was 62.2 years, $50.5 \%$ were female, $89.5 \%$ white, $70 \%(n=140)$ were randomized at Indiana University and 30\% $(n=60)$ at New York University. 
Patients were recruited between 12/11/2017 and 04/18/2018. The trial was stopped when planned enrollment was completed. There was no interim analysis.

Patients screened and excluded were tracked in Indianapolis only. A total of 173 patients were screened there, of whom 27 were excluded including 2 for known severe diverticular disease. The remaining patients were excluded for young age $(n=9)$, referral for resection of a large polyp $(n=8)$, prior colon resection $(n=4)$, inflammatory bowel disease $(n=2)$, Lynch Syndrome $(n=1)$ and polyposis syndrome $(n=1)$. Six screened patients refused participation. A gastroenterology fellow assisted with the insertion of 32 (23\%) cases at Indiana University, and no cases at New York University. Table 1 compares the Endocuff Vision and standard colonoscopy groups by age, gender, race, site, indication, and whether a fellow assisted with insertion and bowel preparation quality. The percentage of females in the standard group (57.6\%) was higher than in the Endocuff Vision group $(43.6 \%) \mathrm{p}=0.048$. There were no other demographic differences between the study arms and none between study sites.

Table 2 shows the inspection and insertion times for the two study arms, after controlling for age, gender, and race. The inspection time using Endocuff Vision was shorter at $6.5 \mathrm{~min}$ compared to 8.4 min with standard colonoscopy $(\mathrm{p}<0.001)$. The absolute mean reduction in inspection time was $23 \%$ (mean 1.93 minutes). Shorter inspection time also reached significance $(\mathrm{p}<0.001)$ for each endoscopist independently (data not shown). Overall withdrawal time (including polypectomy, washing and suctioning time) was more than twice as long when polyps were removed compared to no polyps removed (16.7 $\mathrm{min}$ vs $8.0 \mathrm{~min} ; \mathrm{p}<0.001)$, indicating that polypectomy time was the largest contributor to overall withdrawal time. The mean reduction in overall withdrawal time with Endocuff Vision compared to standard colonoscopy was 1 min but was not statistically significant (15.1 vs. $14.1 \mathrm{~min}$; $\mathrm{p}=0.31)$. In Indianapolis an adult 
colonoscope was used in most cases. In New York a pediatric colonoscope was used in all cases. The cecum was reached in all 200 patients. Insertion time was numerically shorter using Endocuff Vision by 22 seconds, but this difference did not reach significance $(\mathrm{p}=0.143)$.

All detection endpoints numerically favored Endocuff though none of them reached statistical significance. The number of adenomas per colonoscopy (1.43 versus $1.07 ; \mathrm{p}=0.07$ ), adenoma detection rate (61.4\% versus $52 \%$; $\mathrm{p}=0.206)$, sessile serrated polyps per colonoscopy ( 0.27 versus $0.21 ; p=0.120)$, sessile serrated polyp detection rate $(19.8 \%$ versus $11.1 \%$; $\mathrm{p}=0.090$ ), were all numerically higher with Endocuff with similar results after controlling for age, gender, and race. There were no significant differences between the study arms in prevalence of lesions $\geq 10 \mathrm{~mm}$ in size, or between the overall prevalence of any lesion type considered only in the proximal colon (proximal to the splenic flexure) or in the distal colon.

There were no complications in either arm.

Discussion

In this study, we demonstrated that performance of colonoscopy with Endocuff Vision allowed shorter inspection times (23\%), and by an average of almost 2 minutes, with no loss of detection. Because withdrawal times are a continuous variable that are measured in every procedure performed in the study, it's possible to demonstrate statistically significant differences in withdrawal time with considerably smaller sample sizes than are needed to demonstrate detection differences. Despite that, the difference in adenomas per colonoscopy in the current study almost reached statistical significance in favor of Endocuff Vision, and all of the detection endpoints were numerically in favor of Endocuff Vision. $\mathrm{We}^{2}$ and others ${ }^{1}$, have previously demonstrated in what are typically much larger studies, that when inspection times are forced to be equal, Endocuff results in statistically significant gains in detection ${ }^{1,2}$. Although the sample 
size in the current study was not large enough, nor was the study designed to demonstrate both shorter inspection time and superior detection with Endocuff Vision, these data suggest that an appropriately powered study could demonstrate that Endocuff Vision can achieve both improved detection and shorter examination times. The absolute reduction in overall mean withdrawal time with Endocuff Vision, including polypectomy and all washing and suctioning was 1 minute, despite a one-third increase in the number of conventional adenomas and sessile serrated polyps removed in the Endocuff Vision arm.

In our study, more females were randomized to the standard colonoscopy arm than the Endocuff arm, and female gender is associated with a lower prevalence of polyps. Despite that, the numerical trends toward higher detection for all detection endpoints persisted in multivariable analyses that controlled for age, gender, and race.

We found that longer withdrawal times were associated with increasing age and male gender. Since both of these factors are associated with increasing prevalence of adenomas, it's possible that the longer times reflected bias on the part of the investigators, with additional time taken to avoid missing lesions. Alternatively, anatomic factors involved in examining older colons in male patients may account for these differences.

The principle limitation of our study is that the investigators were not blinded to the device used. However, this limitation is common to endoscopic studies, including essentially all studies centered on detection during colonoscopy. Studies of this type depend on the investigators' faithful attempt to minimize bias. Our results have an obvious potential explanation from a mechanistic standpoint, and are consistent with previous uncontrolled studies of Endocuff ${ }^{7}$ and Endorings ${ }^{8}$. Nevertheless, a full picture of the benefits of Endocuff Vision or any device cannot be ascertained without examining multiple studies, because the performance 
of many aspects of colonoscopy, including the use of associated devices, is subject to operator dependence. Because only two endoscopists performed the study, the generalizability of the results remains uncertain. Therefore we encourage other investigators to undertake similar studies. Further, both endoscopists were highly experienced with both Endocuff and Endocuff Vision, and the results may not apply to endoscopists with less experience with the devices. Finally, additional limitations were that we did not collect data on other potential confounders such smoking status and body mass index, and the study was not powered to detect differences in the prevalence of more clinically important lesions such as advanced adenomas or lesions $\geq 10$ $\mathrm{mm}$ in size.

Previous studies, including our own (2), showed that a small fraction of patients have sufficient sigmoid narrowing or angulation to require removal of Endocuff to achieve successful navigation of the sigmoid. In the current study no patient required removal of Endocuff Vision, though two patients at Indianapolis were excluded prior to recruitment for known severe sigmoid diverticular disease. Inclusion of patients 40-49 years old, who have a lower risk of severe diverticular disease compared to older persons, may have contributed to the finding. In general, clinicians should expect a small fraction of patients to require device removal for safe advancement through the sigmoid, and colonoscopy without the device should be considered in patients with known severe sigmoid diverticular disease.

Perspectives on whether the reduced inspection time observed in this trial with Endocuff Vision is clinically important, will depend on individual perspectives on efficiency in endoscopy. For extremely efficient endoscopists, one or two minutes time saving per procedure could be considered a significant gain when many procedures in a day or week are considered.

Efficiency, which should never take place at the expense of quality, is often achieved through the 
accumulation of numerous measures, each of which by itself may have a quantitatively small impact. In any case, there are few situations described in which improvement in efficiency (reduction of inspection time) can be made without loss of detection.

In conclusion, we demonstrated in a randomized controlled trial that Endocuff Vision is associated with reductions in inspection time without loss of detection. We recommend that other investigators examine the impact of Endocuff Vision on inspection and withdrawal times in addition to detection endpoints. 


\section{References}

1. Williet N, Tournier Q, Vernet C, et al. Effect of Endocuff-assisted colonoscopy on adenoma detection rate: meta-analysis of randomized controlled trials. Endoscopy 2018. doi: 10.1055/a-05773500

2. Rex DK, Repici A, Gross SA, et al. High-definition colonoscopy versus Endocuff versus EndoRings versus Full-Spectrum Endoscopy for adenoma detection at colonoscopy: a multicenter randomized trial. Gastrointest Endosc 2018. doi: 10.1016/j.gie.2018.02.043

3. Ngu WS, Bevan R, Tsiamoulos ZP, et al. Improved adenoma detection with Endocuff Vision: the ADENOMA randomised controlled trial. Gut 2018. doi: 10.1136/gutjnl-2017-314889

4. Dik VK, Gralnek IM, Segol O, et al. Multicenter, randomized, tandem evaluation of EndoRings colonoscopy--results of the CLEVER study. Endoscopy 2015;47:1151-8. doi: 10.1055/s-0034-1392421

5. Gralnek IM, Siersema PD, Halpern Z, et al. Standard forward-viewing colonoscopy versus fullspectrum endoscopy: an international, multicentre, randomised, tandem colonoscopy trial. Lancet Oncol 2014;15:353-60. doi: 10.1016/S1470-2045(14)70020-8

6. Rex DK. Colonoscopic withdrawal technique is associated with adenoma miss rates. Gastrointest Endosc 2000;51:33-6. doi: 10.1016/S0016-5107(00)70383-X

7. Tsiamoulos ZP, Misra R, Rameshshanker R, et al. Impact of a new distal attachment on colonoscopy performance in an academic screening center. Gastrointest Endosc 2018;87:280-7. doi: 10.1016/j.gie.2017.04.001

8. Thygesen JC, Ponugoti, P, Tippins, W, et al. Faster colonoscope withdrawal time without impaired detection using EndoRings. Endoscopy International Open 2018;(in press).

9. Leufkens AM, DeMarco DC, Rastogi A, et al. Effect of a retrograde-viewing device on adenoma detection rate during colonoscopy: the TERRACE study. Gastrointest Endosc 2011;73:480-9. doi: 10.1016/j.gie.2010.09.004

10. Barclay RL, Vicari JJ, Doughty AS, et al. Colonoscopic withdrawal times and adenoma detection during screening colonoscopy. N Engl J Med 2006;355:2533-41.

11. Butterly L, Robinson CM, Anderson JC, et al. Serrated and adenomatous polyp detection increases with longer withdrawal time: results from the New Hampshire Colonoscopy Registry. Am J Gastroenterol 2014;109:417-26. doi: 10.1038/ajg.2013.442

12. Shaukat A, Rector TS, Church TR, et al. Longer Withdrawal Time Is Associated With a Reduced Incidence of Interval Cancer After Screening Colonoscopy. Gastroenterology 2015;149:952-7. doi: 10.1053/j.gastro.2015.06.044 
Table 1 Demographics, indications, and number of colonoscope insertions by fellows in the two study arms

\begin{tabular}{|c|c|c|c|}
\hline Demographic & $\begin{array}{c}\text { Endocuff } \\
\text { Vision }\end{array}$ & $\begin{array}{c}\text { Standard } \\
\text { colonoscopy }\end{array}$ & p \\
\hline Screening/surveillance* & $\mathrm{n}=101$ & $\mathrm{n}=99$ & .322 \\
\hline Age (years)** & $45 / 56$ & $52 / 47$ & .446 \\
\hline Female** & $62.7(8.9)$ & $61.7(9.9)$ & .048 \\
\hline White** & $44(43.6)$ & $57(57.6)$ & .117 \\
\hline $\begin{array}{c}\text { Fellow assisting } \\
\text { insertion** }\end{array}$ & $87(86.1)$ & $92(92.9)$ & .949 \\
\hline
\end{tabular}

*number of patients with screening/surveillance indication

**Values are means (standard deviations) for continuous variables and frequencies (percentages) for categorical variables. 
Table 2. Primary and secondary study results.

\begin{tabular}{|c|c|c|c|}
\hline & Endocuff Vision & Standard & p \\
\hline $\begin{array}{c}\text { Inspection time } \\
(\text { min) }\end{array}$ & $\mathrm{n}=101$ & $\mathrm{n}=99$ & $<0.001$ \\
\hline $\begin{array}{c}\text { Insertion time } \\
\text { (min) }\end{array}$ & $4.49(0.10)$ & $4.42(0.12)$ & 0.143 \\
\hline $\begin{array}{c}\text { Boston Bowel } \\
\text { Prep Score } \\
\text { median (range) }\end{array}$ & $9(3-9)$ & $9(3-9)$ & 0.688 \\
\hline $\begin{array}{c}\text { Adenoma } \\
\text { detection rate }\end{array}$ & $61.4 \%(51.6,70.3)$ & $52.5 \%(42.8,62.1)$ & 0.206 \\
\hline $\begin{array}{c}\text { Adenomas per } \\
\text { colonoscopy }\end{array}$ & $1.43(1.79)$ & $1.07(1.62)$ & 0.077 \\
\hline $\begin{array}{c}\text { Sessile serrated } \\
\text { polyp detection } \\
\text { rate }\end{array}$ & $19.8 \%(13.2,28.6)$ & $11.1 \%(6.3,18.8)$ & 0.090 \\
\hline $\begin{array}{c}\text { Sessile serrated } \\
\text { polyps per } \\
\text { colonoscopy }\end{array}$ & $0.27(0.63)$ & $0.21(0.70)$ & 0.120 \\
\hline
\end{tabular}

Values are means (standard errors) for continuous variables, except where noted, and percentages (95\% confidence intervals) for categorical variables. 


\title{
Prospective Randomized controlled trial of High-Definition White- light colonoscopy versus High-Definition White-light colonoscopy with Endocuff Vision for endpoints of procedural times
}

\author{
Primary Investigator:
}

Dr. Douglas Rex, Indiana University Hospital

Indianapolis, IN 46202

\section{Introduction:}

Endocuff Vision ( Olympus) is a disposable device that has 8 independently moving soft flexible arms arranged in a single row. The device comes in several sizes and can fit the tips of most commonly used colonoscopes. During intubation, the arms fall flat to the body of the scope as not to impede forward motion. Endocuff Vision is an FDA approved device that fits over the end of a colonoscope and has flexible soft fingers that project from the sides. These fingers can be used to pull back the folds in the colon and expose mucosa on the proximal sides. A number of studies indicate that Endocuff improves the adenoma detection rate (ADR) during colonoscopy. In a non-randomized retrospective evaluation, Endocuff resulted in better detection, faster insertion, and faster inspection (1).

Our aim in this study is to show in a prospective randomized trial Endocuff Vision could improve insertion because during loop withdrawal the cuff tends to stabilize the position of the tip, preventing its backward movement and speeding loop removal. Similarly, during inspection, the main goal is to evaluate the proximal sides of folds and flexures. The process is made easier and quicker by the device. Third, during polypectomy, Endocuff Vision helps to stabilize the position and the scope tip.

\section{Methods:}

This will be a prospective, randomized controlled study. Subjects referred for screening or surveillance colonoscopy will be prospectively enrolled. They will be randomized to one of two arms, i.e High Definition colonoscopy or High Definition colonoscopy with Endocuff Vision.

A member of the research team will approach a potential subject to discuss participation in the study, including background of the proposed study, inclusion and exclusion criteria, benefits and risks of the procedures and follow-up. If this is of interest to the subject, the informed consent form is discussed and presented. The subject must sign the consent form prior to enrollment. This form will have prior approval of the study site's Institutional Review Board (IRB). Failure to obtain informed consent renders the subject ineligible for the study 


\section{Objectives:}

Primary objectives of the study are:

To compare the insertion time, inspection time, the total time per polypectomy, and the total procedure time during High Definition colonoscopy with Endocuff Vision versus standard High Definition colonoscopy.

Secondary objectives:

To compare the number of adenomas detected per subject with High Definition Endocuff Vision compared to Standard High Definition colonoscopy.

To compare the detection rates for polyp subtypes (including advanced adenomas, serrated polyps, right sided adenomas, etc) for High Definition Endocuff Vision compared to Standard High Definition colonoscopy.

\section{Primary End-point:}

The inspection time will be shorter because the proximal sides of the folds are more quickly exposed with Endocuff Vision compared to standard colonoscope alone because of the ability of the Endocuff Vision to stabilize the position of the colonoscope tip during loop withdrawal, thus preventing its backward movement and speeding loop removal.

\section{Criteria:}

All colonoscopies in the study will be performed by a qualified professional. Patients will be 40 and older and will be randomized to receive colonoscopy with or without the Endocuff. Data collected will include the type of scope used (adult versus pediatric), age, gender, indication, history of prior abdominal surgery and type, identification of diverticulosis classified as mild moderate or severe, insertion time, total procedural time, total withdrawal time, inspection time (withdrawal time minus anytime suctioning, cleaning, and diagnostic biopsy or therapeutic procedure), and polypectomy time for each polyp removed. For each polyp, we will record the location, size, shape, and pathology.

\section{Inclusion criteria}

- Referral for screening or surveillance colonoscopy

- Ability to provide informed consent

- 40 years and older 


\section{Exclusion criteria}

- Prior history of colon cancer

- History of inflammatory bowel disease

- Prior surgical resection of any part of the colon

- Use of anti-platelet agents or anticoagulants that precludes the removal of polyps during the procedure

- History of polyposis syndrome or HNPCC

- Inability to give informed consent

- Family history of colon cancer in a first degree relative $<60$ years or two first degree relatives with colorectal cancer

\section{Statistical Analysis and Sample Size:}

- Normally distributed continuous variables will be summarized using means and standard deviations while non-normally distributed continuous variables will be summarized using medians and ranges.

- The primary endpoints for the study will be the inspection time and insertion time, , the total time per polypectomy, and the total procedure time.

- The secondary endpoints will be total time per polypectomy, total procedure times, and adenomas detected.

- All colonoscopies in the study will be performed by a qualified professional. Patients will be 40 and older and will be randomized to receive colonoscopy with or without the Endocuff. Data collected will include the type of scope used (adult versus pediatric), age, gender, indication, history of prior abdominal surgery and type, identification of diverticulosis (classified as mild moderate or severe), insertion time, total procedural time, total withdrawal time, inspection time (withdrawal time minus anytime suctioning, cleaning, and diagnostic biopsy or therapeutic procedure), and polypectomy time for each polyp removed. For each polyp, we will record the location, size, shape, and pathology.

There will be a sample size of 200 subjects enrolled in this study. 100 randomized into two groups. With the sample size of 100 per group, the study will have $80 \%$ power to detect a 1 minute difference in the inspection time between the two groups. The inspection and insertion times will be compared using a two-sided two sample t-test, 5\% significance level, and standard deviation of 2.5 minutes.

The data will be analyzed and the statistics performed by persons in the biostatistics department. 


\section{Safety Risks to the patient and Data integrity:}

All paper charts pertaining to the patient will be kept under lock and key in coordinators office away from the endoscopy area. The data entry will be performed into an excel file which will be stored on an internal network drive with encryption and password security. Data management will include double data entry and regular back-up of the data. Only approved personnel by the IRB will have access to the file storage. This file will also not have any identifiable information. A study log with the identifiable information will be kept in a separate folder to enable the investigators to assist in any research audit. No procedural data except the date of examination will be entered into this log.

Any subject who wishes to withdraw from this investigation on his/her own accord and for whatever reason is entitled to do so without obligation and prejudice to further treatment. In addition, the Investigator may decide for reasons of medical prudence, to withdraw a subject. In either event, the Investigator will clearly document the date and reason(s) for the subject's withdrawal from this investigation in the CRF and should indicate whether or not he considers it was related to the study interventions.

\section{$\underline{\text { References: }}$}

(1). ZP Tsiamoulos et al. Gastrointest Endosc. 2017 Apr 13. Impact of a New Distal Attachment on Colonoscopy Performance in an Academic Screening Center 
Acronyms

U.S.-United States

vs-versus

ADR-adenoma detection rate

UK-United Kingdom

PA-Pennsylvania

NYU-New York University

GEEs-Generalized Estimating Equations

ANCOVA-analysis of covariance

NC-North Carolina 
In a randomized controlled trial targeted to inspection time during colonoscopy withdrawal, Endocuff Vision was associated with a 1.9 minute reduction in inspection time and numerical increases in all detection targets. 